\title{
Interfacial Electric Phenomena and Donnan Potential in Membranes*1
}

\author{
Hiroyuki Ohshima, Kimiko Makino and Tamotsu Kondo*2 \\ Faculty of Pharmaceutical Sciences and Institute of Colloid and Interface Science \\ Science University of Tokyo, Shinjuku-ku, Tokyo 162
}

(Received, June, 20, 1987)

A model is described for the potential distribution across a charged membrane surface. This model assumes that membrane-fixed charges are distributed through a surface layer of non-zero thickness $d$ and that electrolyte ions can penetrate the surface layer. Along the lines of this model, we discuss various interfacial electric phenomena-potential distribution iacross a membrane, electrophoresis, and electrostatic interaction of membranes - in comparsion with results predicted from the conventional model assuming $d=0$. The Donnan potential is shown to play a fundamental role in the electric behavior of membranes.

Key word: Interfacial electric phenomena, Donnan potential, Electrophoresis, Membrane interaction

\section{Introduction}

The surface charge of biocolloids, which arises from their membrane charges, plays an important role in various interfacial electric phenomena such as motion of cells in an external field (i. e., cell electrophoresis), binding of electrolyte ions to the cell surface and electrostatic interaction of biocolloids (e.g., intercellular interactions, virus-host cell interactions and phagocytosis).

In theories of the electrophoresis and electrostatic interaction of colloid particles, it is usually assumed that the particle charges are located only at the particle surface (of zero thickness) and that the particle surface is ion-impenetrable. However, this assumption is by no means a good approximation to biological membranes.

* 1 膜の界面電気現象とドナン電位 本論文は 1987 年国際膜会議 (1987.6.8,01-OA0801東京)に て発表した。

*2 大島広行, 牧野公子, 近藤 保: 東京理科大学薬学部・界 面科学研究所 $\mathbf{T} 162$ 新宿区市ヶ谷船河原町 12
The membrane-fixed charges are distributed through an ion-penetrable surface layer on the membrane core, as schematically shown in Fig.1. Recently, a number of studies based on a membrane model taking into account the abovementioned surface structure of membranes have been carried out on electrophoresis problems ${ }^{1-6)}$, the potential distribution across $a$ membrane ${ }^{7-11)}$, the electrostatic interaction of membranes ${ }^{12-14)}$ and the membrane potential ${ }^{15)}$. In particular, a membrane model proposed by Ohshima and $\mathrm{Ohki}^{7)}$ smoothly unites two different conceptsthe Gouy-Chapmann double layer potential and the Donnan potential. That is, in the limit of zero thickness of the surface charge layer, this model yields the Gouy-Chapmann double layer potential while in the limit of thick surface charge layer, it gives the Donnan potential distribution. Conventionally, the Donnan potential is considered to be accompanied by a discontinuous potential gap across the membrane surface. However, this is not correct. Due to 


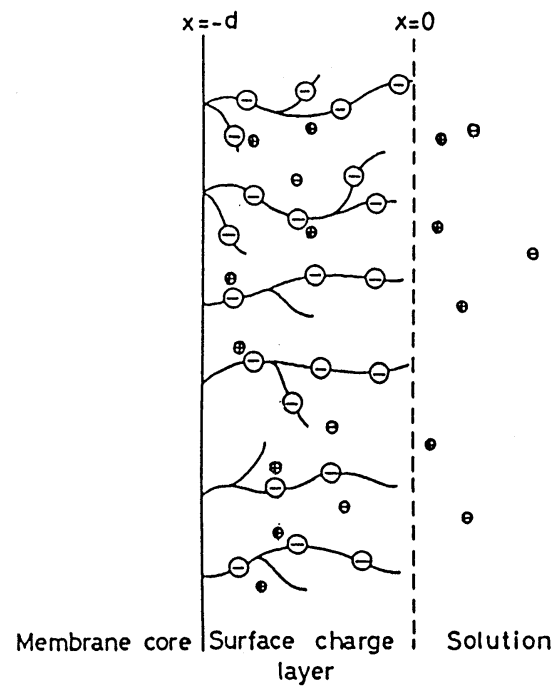

Fig.1 Schematic representation of a membrane with an ion-penetrable surface charge layer of thickness $d$.

Large $\oplus$ and $\Theta$, respectively, stand for mobile cations and anions, and small $\ominus$ for the membrane-fixed charges.

the thermal motion of ions, the potential should diffuse over some distance on both sides of the membrane/solution interface. We shall call this potential distribution the Donnan potential distribution, which includes not only the Donnan potential itself but also the diffusive potential near the interface.

In the present paper we discuss various interfacial electric phenomena in membranes with particular emphasis on an important role played by the Donnan potential distribution.

\section{Potential distribution across a membrane}

Consider a planar charged membrane having an ion-penetrabbe surface charge layer of thickness $d$ immersed in a solution of a symmetrical electrolyte of bulk concentration $n$ and valence $v$. It is assumed that the membrane-fixed negative charges are distributed at a uniform density $N$ in the surface layer. We take the $\mathrm{x}-$ axis perpendicular to the membrane with its origin at the membrane/solution interface (Fig.1).

The Poisson-Boltzmann equations for the po- tential $\psi(x)$ are given by

$$
\begin{aligned}
& \frac{d^{2} \psi}{d x^{2}}=\frac{2 n v e}{\varepsilon_{r} \varepsilon_{0}} \sinh \frac{v e \psi}{k T}, 0<x<+\infty, \\
& \frac{d^{2} \psi}{d x^{2}}=\frac{2 n v e}{\varepsilon_{r} \varepsilon_{0}}\left[\sinh \frac{v e \psi}{k T}+\frac{N}{2 n v}\right],-d<x<0,
\end{aligned}
$$

where $\varepsilon_{r}$ is the relative permittivity of the solution, $\varepsilon_{0}$ is the permittivity of a vacuum, $e$ is the elementary electric charge, $k$ is the Boltzmann constant, and $T$ is the absolute temperature. The second term in the brackets on the right-hand side of Eq. (2) corresponds to the contributiom from the membrane-fixed charges. The boundary conditions are

$$
\begin{aligned}
& \psi(x) \text { is continuous at } x=0 \\
& \psi(x) \rightarrow 0 \text { as } x \rightarrow+\infty \\
& \left.\frac{d \psi}{d x}\right|_{-0}=\left.\frac{d \psi}{d x}\right|_{+0}
\end{aligned}
$$

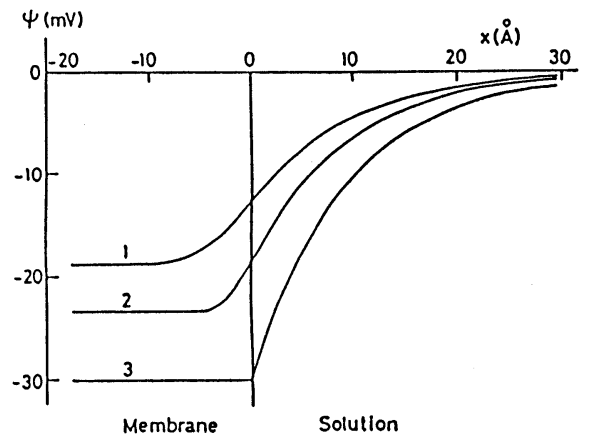

Fig. 2 Potential distribution $\psi(x)$ across membranes with $d=10,5$ and $0 \AA$ (curves 1,2 , and 3 , respectively).

$-e N d=-0.0229 \mathrm{C} / \mathrm{m}^{2}$. $T=298 \mathrm{~K}, v=1$, $n=0.1 \mathrm{M}$

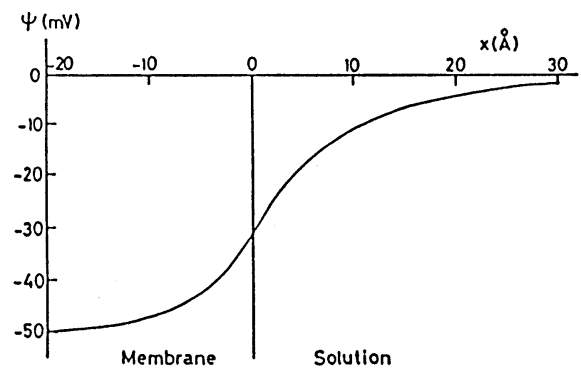

Fig. 3 Potential distribution $\psi(x)$ across a membranes with $d=\infty$. Calculated with $N=0.69 \mathrm{M}, T=298 \mathrm{~K}, v=1, n=0.1 \mathrm{M}$. 
膜 (MEMBRANE), Vol.12 No.7 (1987)

The solution of coupled equations (1) and (2) subject to the boundary conditions (3)-(6) determines the potential distribution of the present system. Examples of the numerical calculation are given in Figures 2 and 3.

For small values of the surface layer thickness $d$, the potential distribution across a membrane strongly depends on $d$, as shown in Fig. 2, which displays numerical results for $\psi(x)$ when $d=0$, 5 , and $10 \AA$, where the product $-e N d$ is kept constant, i.e., the amount of the membrane-fixed charges contained in the surface charge layer per unit area is kept constant. For large $d \gtrsim 1 /$ $x\left[x=\left(2 n v^{2} e^{2} / \varepsilon_{r} \varepsilon_{0} k T\right)^{1 / 2}\right.$ is the Debye-Húckel parameter], however, the potential in the surface layer except in the region very near the membrane/solution interface is almost equal to the Donnan potential, viz.,

$$
\psi_{\mathrm{D}}=-\frac{k T}{v e} \ln \left[\frac{N}{2 v n}+\left\{\left(\frac{N}{2 v n}\right)^{2}+1\right\}^{1 / 2}\right],
$$

which can be obtained by putting the right-hand side of equation (2) to be zero. Note that the Donnan potential thus obtained agrees with the one obtained from the condition of thermo dynamic equilibrium, i.e., by equating the electrochemical potentials of electrolyte ions in the surface charge layer and the solution.

Figure 3 shows the potential distribution for the limiting case of infinite $d$. As stated in the Introduction, we call this the Donnan potential distribution, which includes the diffusive structure of the potential distribution at the membrane/solution interface as well as the Donnan potential itself far inside the membrane. The Donnan potential distribuition can be approximated by

$$
\begin{aligned}
& \psi(x)=\psi_{\mathrm{D}}+\left(\psi(0)-\psi_{\mathrm{D}}\right) \\
& \quad \times \exp \left[K\left\{1+\left(\frac{N}{2 v n}\right)^{2}\right\}^{1 / 4} x\right],-d<x<0
\end{aligned}
$$

where

$$
\begin{aligned}
\psi(0)= & -\frac{k T}{v e}\left(\ln \left[\frac{N}{2 v n}+\left\{\left(\frac{N}{2 v n}\right)^{2}+1\right\}^{1 / 2}\right]\right. \\
& \left.-\left[\left\{\left(\frac{2 v n}{N}\right)^{2}+1\right\}^{1 / 2}-\frac{2 v n}{N}\right]\right)
\end{aligned}
$$

is the membrane surface potential.

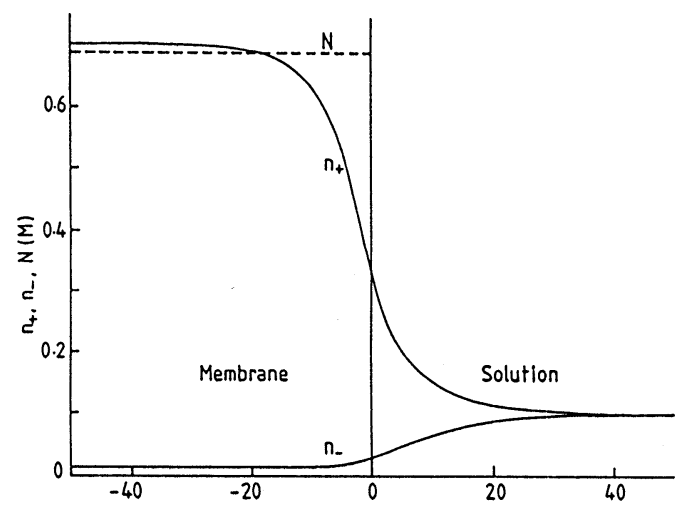

Fig. 4 Distribution of the concentrations of cations $n_{+}(x)$ and anions $n_{-}(x)$ across a membrane with $d=\infty . N=0.69 \mathrm{M}$. $T=298 \mathrm{~K}$, $v=1, n=0.1 \mathrm{M}$.

Figure 4 shows the distribution of cations and anions across the same membrane as in Fig. 3. Far inside the membrane, where the potential is the Donnan potential, the concentration difference between cations and anions equals the density of the membrane-fixed charges $N$ so that the electroneutrality holds.

\section{Electrophoretic mobility}

Now we consider the motion of a particle having a surface charge layer in an external electric field $E$. The fluid velocity $u(\mathrm{x})$ satisfies the Navier-Stokes equations, viz.,

where $\rho_{\text {el }}$ the space charge density resulting from the electrolyte ions, $\eta$ is the viscosity and $r$ is the frictional coeffidient of the surface charge layer. Equations (10) and (11) are subject to the following boundary conditions:

$u=0$ at $x=-d$,

$u$ is continuous at $x=0$,

$$
\left.\frac{d u}{d x}\right|_{-0}=\left.\frac{d u}{d x}\right|_{+0}
$$

$u \rightarrow-U$ as $x \rightarrow+\infty$.

$$
\begin{aligned}
& \eta \frac{d^{2} u}{d x^{2}}+\rho_{\mathrm{el}}(x) E=0,0<x<+\infty, \\
& \eta \frac{d^{2} u}{d x^{2}}-\gamma u+\rho_{\mathrm{el}}(x) E=0,-d<x<0,
\end{aligned}
$$


The electrophoretic mobility $\mu=U / E$ can be obtained by combining the solution of the PoissonBoltzmann equations (1) and (2) with the solution of the Navier-Stokes equations (10) and (11). The result is

$$
\begin{aligned}
\mu & =\frac{\varepsilon_{r} \varepsilon_{0}}{\eta} \frac{1}{\cos \mathrm{h} \lambda \mathrm{d}}[\psi(-d) \\
& \left.+\lambda \int_{-d}^{0} \psi(x) \sinh \lambda(x+d) d x\right] \\
& +\frac{\sigma}{\eta d \lambda^{2}}\left(1-\frac{1}{\cosh \lambda d}\right)
\end{aligned}
$$

with

$$
\begin{aligned}
& \lambda=(r / \eta)^{1 / 2}, \\
& \sigma=-e N d .
\end{aligned}
$$

As these equations show, the electrophoretic mobility depends on the whole potential distribution across the membrane surface charge layer.

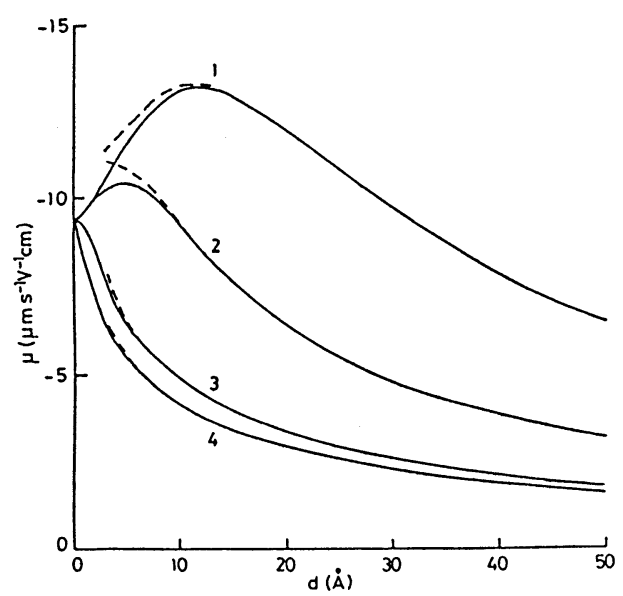

Fig.5 The electrophoretic mobility $\mu$ as a function of the surface layer thickness $d$ for several values of $\lambda$.

Curve $1, \lambda=1 \times 10^{9} \mathrm{~m}^{-1}$; Curve $2, \lambda=2 \times 10^{9} \mathrm{~m}^{-1}$. Curve $3, \lambda=1 \times 10^{10} \mathrm{~m}^{-1}$; Curve $4, \lambda=\infty$.

Calculated with $T=298 \mathrm{~K}, v=1, n=0.1 \mathrm{M}$, $\eta=0.891 \times 10^{-3} \mathrm{Nsm}^{-2}$ and $-e N d=-0.19 \mathrm{C} / \mathrm{m}^{2}$. - exact results (Eq. (16)) : ---approximation (Eq. (8)).

In Fig. 5, we display the mobility as a function of the surface layer thickness $d$ for several values of $\lambda$, where $-e N d$ is kept constant. The value at $d=0$ corresponds to the wellknown Smolchowski formula, viz..

$$
\mu=\frac{\varepsilon_{r} \varepsilon_{0}}{\eta} \psi(0)
$$

Figure 5 shows that in some cases the mobility curve has a maximum (curves 1 and 2). This behavior is due to the second term of Eq. (16), which increases linearly with increasing $d$ for small $d$ and then decreases as $1 / d$ for large $d$. The increase in $\mu$ at small $d$ is due to the increase in contribution from the portion of the membrane-fixed charges that lies away from the plane $x=-d$, at which the fluid velocity is zero. Note that the second term does not depend on the electrolyte concentration. The first term, on the other hand, depends on the electrolyte concentration and decreases as $d$ increases. The above-mentioned opposite dependence of the mobility can in some cases exhibit a maximum. Figure 5 also shows the approximate results calculated by using the Donnan potential distribution (8), which are seen to be in fairly good agreement with the exact results.

\section{Electostatic interaction of two membranes}

The electrostatic interaction between membranes with surface charge layers also depends on the potential distribution in the surface charge layers of the membranes.

The electrostatic repulsion (double-layer repulsion) $P(h)$ per unit area between two membranes at separation $h$ can be obtained by integrating Maxwell's stress and the osmotic pressure over an arbitrary surface enclosing either one of the two membranes. If the potential is sufficiently low, linearization of the Poisson-Boltzmann equations gives

$$
P(h)=\frac{1}{2} \varepsilon_{r} \varepsilon_{0} \kappa^{2} \psi^{2}(h / 2)
$$

where $\psi(h / 2)$ is the potential at the mid-plane between the membranes. As has already been shown, if the surface layer thickness $d \gtrless 1 / x$, the potential distribution is almost equal to that for $d=\infty$, where the potential far inside the membrane is the Donnan potential. Since the 
Donnan potential is determined solely by the thermodynamic equilibrium condition, the potential far inside the interacting membranes is held constant independent of membrane separation $h$. Under such boundary conditions, integration of Eq. (20) yields the interaction energy $V(h)$ per unit area,

$$
V(h)=2 \varepsilon_{r} \varepsilon_{0} \kappa \psi_{0 e}^{2-x h}
$$

where $\psi_{0}$ is the membrane surface potential of a single non-interacting membran for $h=\infty$.

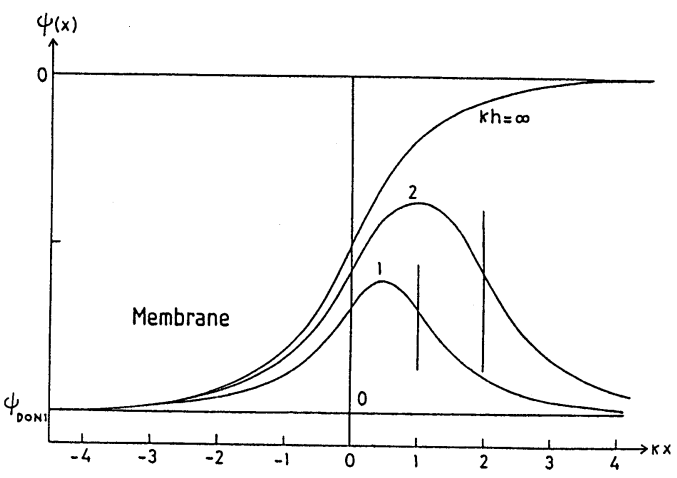

Fig. 6 Change in potential distribution between two interacting ion-penetrable membranes with $d=\infty$ due to their approach.

Figure 6 shows the change in potential distribution between two interacting membranes due to their approach, indicating that the rise in potential in the membranes caused by their approach is greatly supressed so that the potential in the membrane does not exceed the Donnan potential. Figure 7 compares the present model with other two models for the double layer interaction, that is, constant surface potential model $^{16)}$ and constant surface charge density model $^{17)}$, which are given by

$$
V(h)=2 \varepsilon_{r} \varepsilon_{0} \kappa \psi_{0}^{2} \frac{1}{e^{\kappa h}+1}
$$

(Constant surface potential model),

$$
V(h)=2 \varepsilon_{r} \varepsilon_{0} \kappa \psi_{0}^{2} \frac{1}{e^{\kappa h}-1}
$$

(Constant surface charge density model).

In the first model the surface potential remains unchanged while in the second model the surface

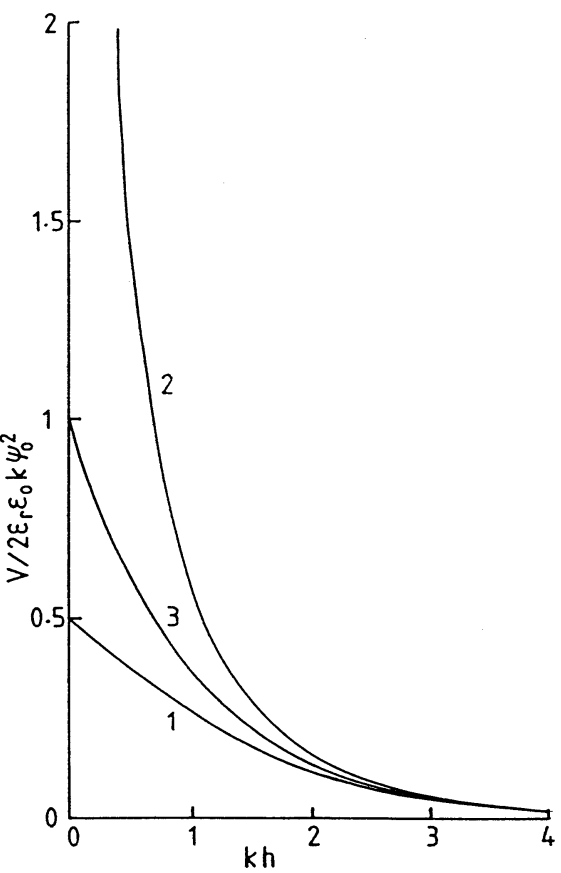

Fig. 7 Comparison of three models on the double-layer intraction. Curve 1 , constant surface potential ; curve 2, constant surface charge density; curve 3 , Donnan potential regulation $(d=\infty)$.

potential increases remarkably during interaction. It is seen that the present model, which may be called the Donnan potential regulation model, gives values of repulsion that lie between those predicted by the other two models.

\section{References}

1) E. Donath, V. Pastushenko: Bioelectrochem. Bioenerg., 6, 543 (1979)

2) R. W. Wunderlich: J. Colloid Interface Sci., 88, 385 (1982)

3) S. Levine, M. Levine, K. A. Sharp, D. E. Brooks: Biophys. J., 42, 127 (1983)

4) K. A. Sharp, D. E. Brooks: Biophys. J., 47, 563 (1985)

5) H. Ohshima, T. Kondo : Colloid Polym. Sci., 264, 1080 (1986)

6) H. Ohshima, T. Kondo: J. Colloid Interface Sci., 116, 305 (1987)

7) H, Ohshima, S. Ohki : Biophys. J., 47, 673 (1985)

8) H. Ohshima, K. Makino, T. Kondo: J. Colloid Interface Sci., 113, 369 (1986)

9) H. Ohshima, T. Kondo: J. theor. Biol., 124, 
430 Ohshima, Makino, Kondo : Interfacial Electric Phenomena and Donnan Potential

191 (1987)

10) K. Makino, H. Ohshima, T. Kondo : J. theor. Biol., 125, 367 (1987)

11) K. Makino, H. Ohshima, T. Kondo: Colloid Polym. Sci., 265, 336 (1987)

12) H. Ohshima, K. Makino, T. Kondo : J. Colloid Interface Sci., 116, 196 (1987)

13) K. Makino, H. Ohshima, T. Kondo: Colloid Polym. Sci., 265, 911 (1987)
14) H. Ohshima, T. Kondo: J. theor. Biol., 128, 187 (1987)

15) H. Ohshima, T. Kondo: Biophys. Chem., in press.

16) E. J. W. Verway, J. Th. G. Overbeek: "Theory of the Stability of Lyophobic Colloids" Elsevier, Amsterdam (1948)

17) H. Ohshima : Colloid Polym. Sci., 252, 158 (1974) 